\title{
Neutrino Emission from Stellar Collapse including Hadron-Quark Mixed Phase
}

\author{
Ken'ichiro Nakazato* \\ Department of Physics, Waseda University, 3-4-1 Okubo, Shinjuku, Tokyo 169-8555, Japan \\ E-mail: nakazato@heap.phys.waseda.ac.jp
}

Kohsuke Sumiyoshi

Numazu College of Technology, Ooka 3600, Numazu, Shizuoka 410-8501, Japan

E-mail: sumi@numazu-ct.ac.jp

\section{Shoichi Yamada ${ }^{\dagger}$}

Department of Physics, Waseda University, 3-4-1 Okubo, Shinjuku, Tokyo 169-8555, Japan

E-mail: shoichi@waseda.jp

\begin{abstract}
Recently, the dynamics and neutrino emission of a black-hole forming stellar collapse is suggested to depend crucially on the equation of state (EOS). In such a case, density and temperature of the matter may become enough high to occur a deconfinement transition of hadronic matter to quark matter. In this study, we perform the gravitational collapse of massive stars using an EOS with the hadron-quark phase transition for finite temperature. In the computations, neutrino transfer equations are solved simultaneously with general relativistic hydrodynamics under spherical symmetry.

A progenitor model with 40 solar masses $\left(M_{\odot}\right)$ is adopted as the initial condition of the collapse. This model has already been shown to produce a bounce before black hole formation. We find that, in this process, the interval time from the bounce to the black hole formation becomes shorter because the transition makes EOS softer. We also find a somewhat unfamiliar decrease of temperature throughout the transition. The event number of neutrinos emitted from our model is evaluated for the currently operating neutrino detector, SuperKamiokande III (SK III). As a result, the total neutrino event number becomes smaller due to the transition. We implied that the sign of the quark appearance can be distinguished by the total event number and energy spectrum.
\end{abstract}

10th Symposium on Nuclei in the Cosmos

July 27 - August 12008

Mackinac Island, Michigan, USA

\footnotetext{
* Speaker.

${ }^{\dagger}$ Also at Advanced Research Institute for Science \& Engineering, Waseda University, 3-4-1 Okubo, Shinjuku, Tokyo 169-8555, Japan.
} 


\section{Introduction}

Hadronic matter undergoes a deconfinement transition to quark matter at high temperature and/or high density. Recently, quark transition is suggested to play important roles in high-energy astrophysical phenomena. In this study, we perform the numerical simulation of the stellar collapse using an equation of state (EOS) including the hadron-quark phase transition. We also evaluate the neutrino event number for the currently operating neutrino detector, SuperKamiokande III (SK III). Further details of this study are seen in our latest paper [1] and work in progress [2].

\section{Numerical Simulation of Stellar Collapse including Quarks}

In this study, we adopt the EOS by Ref. [1], which includes the hadron-quark phase transition in finite temperature. For the hadronic phase, it utilizes a table by Ref. [3] (Shen EOS) based on the relativistic mean field theory. The MIT bag model of the deconfined 3-flavor strange quark matter [4] is used for the quark phase and the hadron-quark mixed phase is obtained by the Gibbs conditions in the EOS. We adopt $B=250 \mathrm{MeV} \mathrm{fm}^{-3}\left(B^{1 / 4}=209 \mathrm{MeV}\right)$ for the bag constant here. As the initial models of our simulation, we choose a stellar model with $40 M_{\odot}$ [5] adopted in Ref. [6]. In this reference, a numerical simulation of the stellar collapse has been already done under the Shen EOS and we follow the scheme of it. We solve the general relativistic hydrodynamics and neutrino transfer equations simultaneously under spherical symmetry. Four species of neutrinos, namely $v_{e}$, $\bar{v}_{e}, v_{\mu}$ and $\bar{v}_{\mu}$, are considered assuming that $v_{\tau}$ and $\bar{v}_{\tau}$ are the same as $v_{\mu}$ and $\bar{v}_{\mu}$, respectively. It is noted that the luminosities and spectra of $v_{\mu}$ and $\bar{v}_{\mu}$ are almost identical because they have the same reactions and the difference of coupling constants is minor.

The radial trajectories of mass elements are shown in Fig. 1a. We can see that the stellar core is bounced once and recollapses to a black hole finally. In this process, the phase transition makes the interval time from the bounce to the black hole formation shorter because the EOS gets softer and the maximum mass of the compact stars becomes smaller owing to the transition. The duration time for the case with the transition is $1.11 \mathrm{~s}$ while that for the case without the transition is $1.35 \mathrm{~s}$. The evolution of the central density and temperature is plotted with the phase diagram in Fig. 1b. The phase diagram is shown for matter with the electron-type lepton fraction $Y_{l}=0.3$, which is almost the same with the value at the center. From this figure we can see that the temperature decreases in the mixed phase despite the density increases. It looks unfamiliar, however, we can interpret it in the context of phase transition [7]. The phase transition dealed in our EOS involves the release of latent heat and the hadronic phase has the lower entropy than the quark phase. On the other hand, matter in the collapsing star is compressed adiabatically. Therefore, the entropy does not vary and the temperature decreases. It is noted that the low-density phase usually has the higher entropy as is the case for a liquid-vapor transition of $\mathrm{H}_{2} \mathrm{O}$.

\section{Evaluation of Neutrino Event Numbers}

Enormous neutrinos are emitted from the stellar collapse. Since the duration of neutrino emission is almost same as the interval time from the bounce to the black hole formation [6], the quark deconfinement reduces the amount of emitted neutrinos. In this section, we evaluate the neutrino 

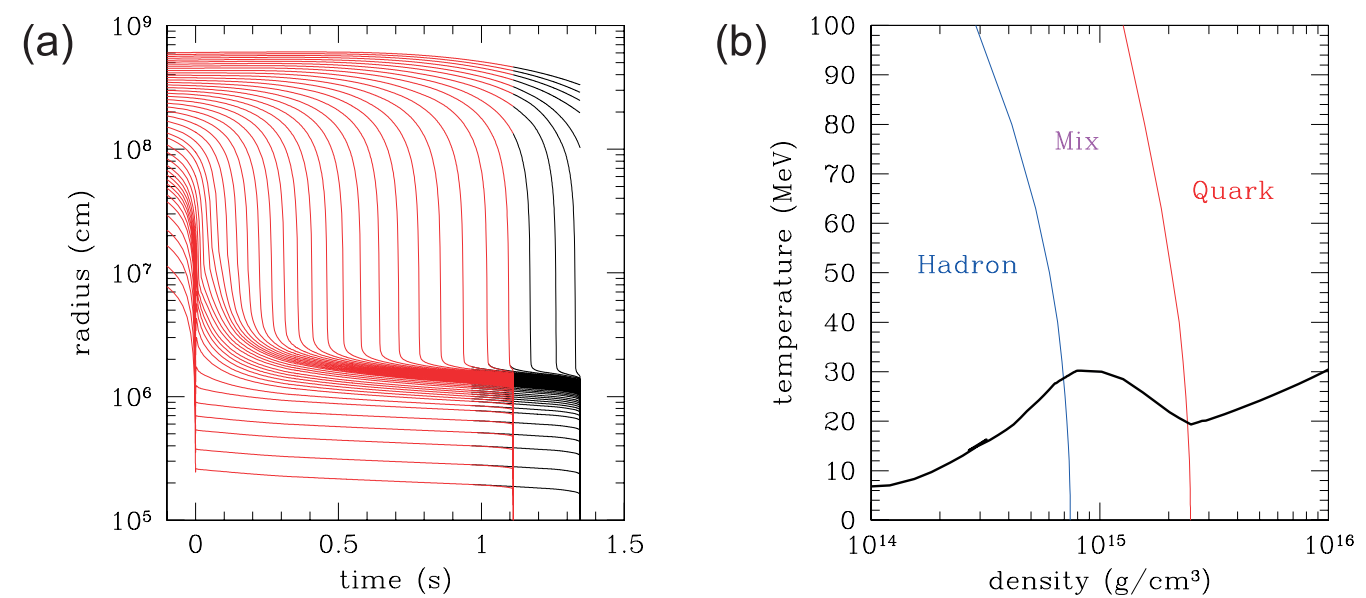

Figure 1: Results of the numerical simulation of stellar collapse. (a): Radial trajectories of mass elements (red lines) with those of the result for the collapse under the Shen EOS in Ref. [6] (black lines). Time is measured from the bounce. $(b)$ : Evolution of the central density and temperature (thick black line) with the phase diagram for $Y_{l}=0.3$. Blue line represents boundary of hadronic matter and mixed matter and red line does that of mixed matter and quark matter.

event number for SK III, taking into account the neutrino oscillation. It is note that, in our case, the black hole formation occurs just after the transition and features of neutrino emission before the black hole formation are almost same with that for the case without the transition in Ref. [6].

The existence of neutrino oscillation is confirmed so far and we should take into account it to evaluate the event numbers. Neutrinos from the stellar collapse propagate through the stellar envelope, where neutrino flavor conversion occurs by the Mikheyev-Smirnov-Wolfenstein effect. When neutrinos pass through the earth before detection, they undergo the flavor conversion also inside the earth. In this case, results of flavor conversions depend also on the nadir angle of the progenitor, which we examine its dependence. In this study, we use the realistic profiles of the progenitor [5] and the earth [8]. As for the parameters of the neutrino mixing, only the upper limit is given for the mixing angle $\theta_{13}$ as $\sin ^{2} \theta_{13} \leq 2.0 \times 10^{-2}$, while the other mixing angles are well measured. Whether the sign of the mass squared difference $\Delta m_{31}^{2}$ is plus (normal mass hierarchy) or minus (inverted mass hierarchy) is also unclear under the current status [9]. Therefore, we also investigate their dependences quantitatively. In this study, we set a distance from a black hole progenitor $R=10 \mathrm{kpc}$, which is typical length of our Galaxy. As for the method to compute the neutrino survival probabilities and event numbers at SK III, we follow Ref. [10].

In Fig. 2, the time-integrated event numbers of the collapse with the hadron-quark phase transition are shown for various parameter sets comparing with those of the case without quarks (the original Shen EOS) in Ref. [6]. Error bars represent the upper and lower limits owing to the different nadir angles. In the case with the earth effects, the energy spectral shape is deformed to wave-like (not shown here). This is because the typical length of neutrino oscillation becomes comparable to the size of the earth and the neutrino survival probabilities become sensitive to the neutrino energy. However, integrating over the neutrino energy, the fluctuation is smoothed out and its impact on the total event number is not so large as seen in Fig. 2. The neutrino event numbers 

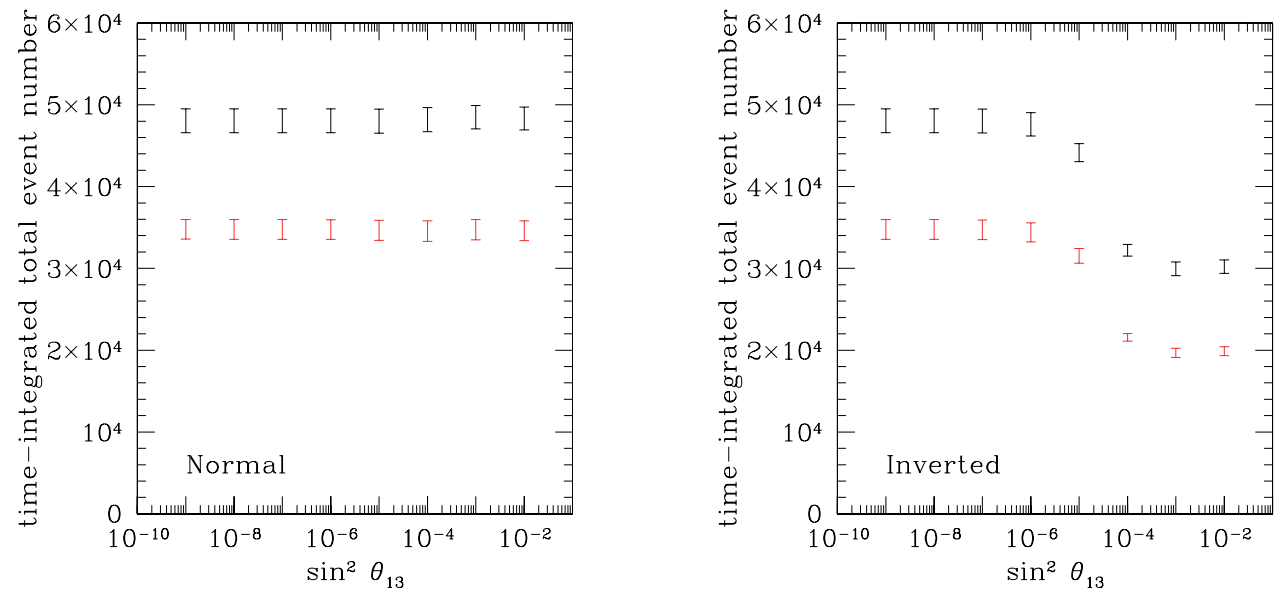

Figure 2: Time-integrated neutrino event numbers for the normal mass hierarchy (left) and the inverted mass hierarchy (right). Error bars represent the upper and lower limits owing to the different nadir angles. Red (lower) sets represent our model including the effects of quarks while black (upper) sets do the model for the original Shen EOS in Ref. [6]

of the case with the quark transition is $\gtrsim 30 \%$ smaller than that of the case without the transition. This difference arises not only from the short duration time of the neutrino emission but also from the low average neutrino energy of the case with quarks. In fact, the duration time for the case with quarks is $\lesssim 20 \%$ shorter than that for the case without quarks. For the collapse involving the black hole formation, the average neutrino energy gets higher for the late phase (see Fig. 15 in Ref. [6]).

We can find that the event number gets smaller for the inverted mass hierarchy with larger $\sin ^{2} \theta_{13}$ from Fig. 2. The difference in the mixing parameters is notably seen in Fig. 3 which shows the energy spectra of the time-integrated event number. The event number for the case of the inverted mass hierarchy with $\sin ^{2} \theta_{13}=10^{-2}$ is much smaller than that for the case of the normal mass hierarchy with $\sin ^{2} \theta_{13}=10^{-8}$, especially for the low energy regime and accordingly the spectrum becomes harder. Unless an ambiguity on the mixing parameters is removed, the neutrino event of the collapse with the quark transition is difficult to be distinguished from that of the collapse without the transition, judging only from the total event number. However, the distinction is implied to be possible by the difference in the spectral feature. The restrictions of the mixing parameters may also be possible by the spectral feature.

\section{Conclusion and Future Work}

In this study, we have shown that the hadron-quark phase transition occurs when the massive star with $40 M_{\odot}$ collapses to the black hole, performing the numerical simulation. Since enormous neutrinos are emitted from this collapse, we have also evaluated the event number for SK III. For the computations, we have taken into account the effect of the neutrino oscillation examining the dependences on the mixing parameters and the nadir angle of the progenitor. The quark transition makes the total neutrino event number smaller because the duration time of the neutrino emission becomes shorter. Additionally, the average neutrino energy becomes lower by the shortening of 

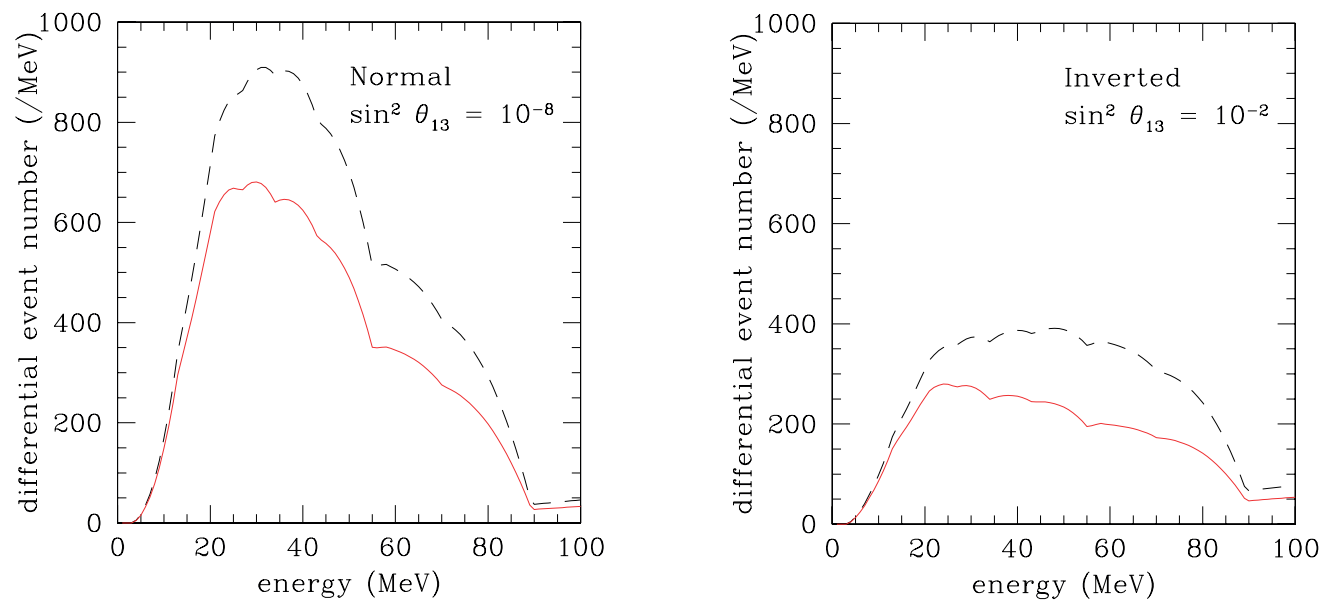

Figure 3: Energy spectra for the time-integrated event number of neutrinos for the normal mass hierarchy with $\sin ^{2} \theta_{13}=10^{-8}$ (left) and the inverted mass hierarchy with $\sin ^{2} \theta_{13}=10^{-2}$ (right). Red solid lines represent our model including the effects of quarks while black dashed lines do the model for the original Shen EOS in Ref. [6]

the duration. Combining this fact with the feature of energy spectrum of detected neutrinos, it is implied that we can probe observationally the EOS of hot and dense matter in future.

However, an ambiguity on the progenitor model still [11]. The appearances of pions [1] and hyperons [12] also affect the neutrino signals. Obviously, more detailed investigations are necessary to probe the EOS of hot and dense matter from neutrino signals. This study is one of the early stages of the following comprehensive investigations.

\section{References}

[1] K. Nakazato, K. Sumiyoshi, and S. Yamada, PRD 77 (2008a) 103006.

[2] K. Nakazato, K. Sumiyoshi, and S. Yamada, (2008b) in prep.

[3] H. Shen, H. Toki, K. Oyamatsu and K. Sumiyoshi, PTP 100 (1998) 1013.

H. Shen, H. Toki, K. Oyamatsu and K. Sumiyoshi, NPA 637 (1998) 435.

[4] A. Chodos, R. L. Jaffe, K. Johnson, C. B. Thorn, and V. F. Weisskopf, PRD 9 (1974) 3471.

[5] S. E. Woosley, and T. Weaver, ApJS 101 (1995) 181.

[6] K. Sumiyoshi, S. Yamada, and H. Suzuki, ApJ 667 (2007) 382.

[7] H. Müller, NPA 618 (1997) 349.

[8] A. M. Dziwonski, and D. L. Anderson, Phys. Earth. Planet. Inter. 25 (1981) 297.

[9] M. C. Gonzalez-Garcia, and M. Maltoni, Phys. Rept. 460 (2008) 1.

[10] K. Nakazato, K. Sumiyoshi, H Suzuki, and S. Yamada, (2008c) in prep.

[11] K. Sumiyoshi, S. Yamada, and H Suzuki, ApJ (2008a) in press, arXiv: 0808.0384 [astro-ph].

[12] K. Sumiyoshi, C. Ishizuka, A. Ohnishi, S. Yamada, and H Suzuki, (2008b) submitted. 